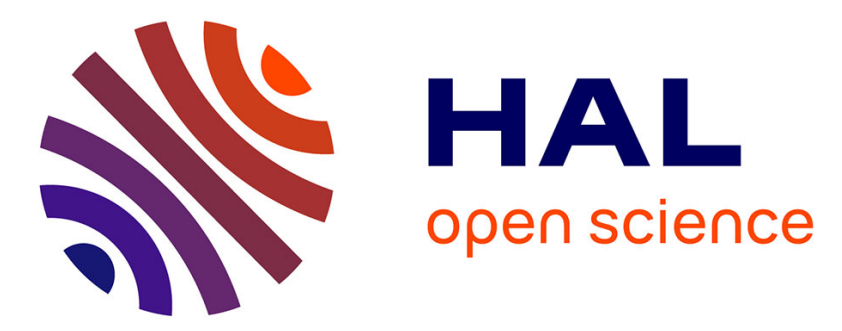

\title{
Les combattants francs et slaves face à la paix : crise et nouvelle définition d'une élite dans l'espace oriental carolingien au début du IXe siècle
}

Thomas Lienhard

\section{- To cite this version:}

Thomas Lienhard. Les combattants francs et slaves face à la paix : crise et nouvelle définition d'une élite dans l'espace oriental carolingien au début du IXe siècle. Les élites au haut Moyen Age. Crises et renouvellements, Brepols, pp.253-266, 2006. hal-03310081

\section{HAL Id: hal-03310081 \\ https://hal.science/hal-03310081}

Submitted on 22 Sep 2021

HAL is a multi-disciplinary open access archive for the deposit and dissemination of scientific research documents, whether they are published or not. The documents may come from teaching and research institutions in France or abroad, or from public or private research centers.
L'archive ouverte pluridisciplinaire HAL, est destinée au dépôt et à la diffusion de documents scientifiques de niveau recherche, publiés ou non, émanant des établissements d'enseignement et de recherche français ou étrangers, des laboratoires publics ou privés. 


\section{(Préprint ! Epreuves non relues !)}

\section{Les combattants francs et slaves face à la paix : crise et nouvelle définition d'une élite dans l'espace oriental carolingien au début du $\mathrm{IX}^{\mathrm{e}}$ siècle}

\section{Thomas Lienhard}

Parmi les nombreux facteurs qui peuvent susciter l'ascension d'une élite ou au contraire, sa chute, il semble évident que la guerre tient une place fondamentale. En premier lieu en effet, si l'on considère une société donnée avec ses usages sociaux en temps de paix, le déclenchement d'un conflit constitue un facteur bouleversant qui contraindra les élites à s'adapter ou à disparaître ; ce point étant évoqué par plusieurs auteurs dans le présent recueil, point n'est besoin d'y revenir ici. Mais en second lieu, il peut être fructueux également d'analyser les évolutions qui surviennent à un stade plus avancé du processus guerrier : il peut arriver, en effet, que la guerre, passé le premier choc, devienne à son tour une situation normale. Celle-ci engendre alors ses propres règles, et par là même de nouveaux critères pour la définition des élites ; dans ce cas, c'est le retour à la paix qui peut constituer un facteur de crise pour ces groupes sociaux. C'est un tel phénomène d'inversion que l'on peut observer dans le dossier proposé ici.

Celui-ci se compose de quelques extraits des Annales du Royaume des Francs pour la période comprise entre 818 et $828^{1}$. Ces textes concernent une situation de conflit, celle qui opposa les armées carolingiennes et les peuples slaves situés au sud-est du monde franc: L'historien est donc confronté là à des sociétés frontalières, caractérisées par une forte mobilité sociale au début du $\mathrm{IX}^{\mathrm{e}}$ siècle. Dans ce contexte, en effet, la constitution ou la disparition des élites était accélérée par la dissolution de l'empire avar, et inversement, par l'expansion récente du pouvoir franc: celui-ci était amené à s'imposer loin de ses assises neustriennes et austrasiennes, et à revoir pour cela le statut des divers groupes en présence. Quel rôle la guerre joua-t'elle dans ces processus ? Il ne sera sans doute pas inutile de présenter d'abord l'interprétation traditionnelle de l'historiographie consacrée à ce conflit, ce qui permettra de camper en même temps le contexte politique et les principaux protagonistes parmi les élites concernées ; on s'efforcera ensuite de démontrer que les élites en question, ou du moins une partie d'entre elles, ont délibérément aggravé le conflit pour préserver leurs intérêts de groupe ; cette hypothèse exigera d'expliquer enfin quels étaient ces intérêts, et de quelle manière un retour à la paix aurait constitué une crise pour cette catégorie des dominants.

L'historiographie explique généralement ces conflits en les présentant comme le difficile accouchement de peuples nouveaux dans ces espaces frontaliers ${ }^{2}$ : on se trouvait en effet dans le contexte d'une vacance politique en Europe centrale, après la chute de l'ensemble avar vers $796^{3}$. En relation avec cet événement, et peut-être également parce que nos sources se firent alors plus précises à propos de cet espace, des peuples nouveaux, ou tombés dans l'oubli

\footnotetext{
${ }^{1}$ Annales Regni Francorum, éd. Friedrich Kurze, MGH Scriptores rerum Germanicarum in usum scholarum 6, Hanovre, 1895 (désormais : ARF). Le lecteur trouvera une traduction de ces extraits à la fin du présent article.

${ }^{2}$ Voir notamment Herwig Wolfram, Die Geburt Mitteleuropas : Geschichte Österreichs vor seiner Entstehung, Vienne, 1987, p. 269, qui évoque une " ethnogénèse embryonnaire ", ou encore Radoslav Katičić, "Die Anfänge des kroatischen Staates", dans Andreas Schwarcz et Herwig Wolfram dir., Die Bayern und ihre Nachbarn. Teil 1. Berichte des Symposions der Kommission für Frühmittelalterforschung, 25. bis 28. Oktober 1982, Stift Zwettl, Niederösterreich, Vienne, 1985 (Veröffentlichungen der Kommission für Frühmittelalterforschung 8), p. 299-312, ici p. 300 sq.

${ }^{3}$ Pour l'analyse de ce phénomène, l'œuvre maîtresse reste celle de Walter Pohl, Die Awaren. Ein Steppenvolk in Mitteleuropa, 567-822 n. Chr., Munich, 1988.
} 
depuis longtemps, furent mentionnés au début du $\mathrm{IX}^{\mathrm{e}}$ siècle par les textes latins consacrés à ces marges orientales. Chez les Slaves du Nord-Ouest, ce fut le cas des Abodrites, mentionnés pour la première fois en $789^{4}$ et occupant ensuite une place croissante dans les textes latins à partir des années 810 ; ce fut surtout le cas des Moraves, apparus en $822^{5}$ et appelés à devenir le principal vis-à-vis oriental des Carolingiens au cours du IX ${ }^{\mathrm{e}}$ siècle $^{6}$; au sud-est de l'empire, face au Frioul, les annalistes nommèrent désormais les Serbes ${ }^{7}$, ou encore des groupes plus éphémères, tels que les Timociani ${ }^{8}$, en attendant la première mention des Croates vers la fin du IX ${ }^{\mathrm{e}}$ siècle ${ }^{9}$. Enfin, un dernier ensemble s'imposa avec éclat parmi ces groupes slaves : il s'agissait des Bulgares, dont le nom avait pourtant disparu des sources depuis l'époque du Pseudo-Frédégaire ${ }^{10}$. Ceux-ci apparurent dans les Annales royales en 812, puis remportèrent une série de victoires militaires à la fois contre Byzance et contre les Carolingiens ${ }^{11}$, apparaissant désormais comme la puissance dominante parmi les Slaves de Pannonie et des Balkans.

L'heure était ainsi à une rapide recomposition ethnique, et cela accéléra la définition ou, au contraire, la disparition des élites. Tel fut le cas à l'extérieur du monde carolingien, où des princes d'ampleur régionale se substituèrent progressivement aux dirigeants de l'espace avar $^{12}$; mais du côté franc également, ces secteurs frontaliers virent les structures de pouvoir antérieures remises en question. La première rupture fut celle d'un renouveau des équipes dirigeantes: avec les conquêtes carolingiennes au Frioul puis en Pannonie, une place croissante fut accordée à des responsables d'origine franque dans ces espaces. Il est vrai que ce renouvellement ne fut pas total: en Pannonie par exemple, intégrée dans le monde carolingien en 796 , le début du $\mathrm{IX}^{\mathrm{e}}$ siècle vit coexister des dirigeants locaux slaves, continuant à gérer les mêmes espaces qu'auparavant, et des nouveaux venus issus du monde franc ${ }^{13}$. Simultanément, par-delà les questions de personnes ou d'origine ethnique, l'ampleur géographique des pouvoir distribués connut elle aussi des variations considérables et parfois très rapides. C'est ainsi notamment qu'en 828, si l'on en croit les Annales, “la marche que

\footnotetext{
${ }^{4}$ ARF, a. 789, p. 84.

5 ARF, a. 822, p. 159.

${ }^{6}$ Voir notamment Gerd Althoff, “Zur Bedeutung der Bündnisse Svatopulks von Mähren mit Franken ”, dans Klaus Trost et al. dir., Symposium Methodianum: Beiträge der internationalen Tagung in Regensburg (1. bis 24. April 1985) zum Gedenken an den 1100. Todestag des hl. Method, Neuried, 1988, p. 13-21, ou plus récemment, l'ouvrage de Martin Eggers, Das „Großmährische Reich“. Realität oder Fiktion? Eine Neuinterpretation der Quellen zur Geschichte des mittleren Donauraumes im 9. Jahrhundert, Stuttgart, 1995 (Monographien zur Geschichte des Mittelalters 40) ; précisons toutefois que ce dernier ouvrage, qui avance une hypothèse originale à propos de la localisation de la Moravie, est très contesté par l'historiographie.

7 ARF, a. 822, p. 159.

${ }^{8}$ Ce peuple est nommé pour la première fois dans les ARF, a. 818, p. 149 ; pour les autres références, on pourra se reporter au dossier traduit en fin du présent article.

${ }^{9}$ On considère généralement que la plus ancienne attestation de cet ethnonyme est épigraphique : il s'agirait d'un fragment trouvé sur la côte dalmate, généralement daté des années 870 : Vedrana Delonga éd., Latinski epigrafički spomenici u ranosrednjovjekovnoj Hrvatskoj (Les monuments épigraphiques latins de la Croatie du Haut Moyen-Age), Split, 1996, n 130. Mais si l'on écarte ce document, dont la datation ne va pas sans difficulté, la première apparition connue du nom de Croates remonterait à l'année 925, avec la correspondance du pape Jean X avec plusieurs destinataires de la côte dalmate : M. Kostrencic et al. éd., Codex diplomaticus regni Croatiae, Dalmatiae et Slavoniae, vol. 1 : Diplomata annorum 743-1100, Zagreb, 1967.

${ }^{10}$ Ils étaient encore nommés, en effet, dans la Chronique de ce dernier, rédigée vers 660 : Frédégaire,

Chronicarum libri IV cum continuationibus, éd. Bruno Krusch, MGH Scriptores rerum Germanicarum in usum scholarum 2, Hanovre, 1888, livre IV, c. 72, p. 157.

${ }^{11}$ Pour le détail de ces événements, on pourra se reporter au dossier de traductions proposé plus loin dans le présent article.

${ }^{12}$ Il est frappant de constater, en particulier, que la première mention des Moraves, en 822, coïncide avec la dernière des Avars.

${ }^{13}$ Conversio Bagoariorum et Carantanorum, éd. Fritz Lošek, Hanovre, 1997 (MGH Studien und Texte 15), c. 10.
} 
possédait seul le duc de Frioul fut divisée en quatre comtés" : l'objectif recherché consistait sans doute à rendre plus efficace la défense contre les Bulgares ${ }^{14}$, mais on amoindrissait également, par la même occasion, la puissance d'un aristocrate franc. Dans ce contexte fluctuant, il s'agissait donc de définir à la fois l'identité ethnique des grands et le pouvoir réel accordé à ces derniers.

Qui put s'imposer dans ce climat d'extrême mobilité sociale? Au sein des groupes francs et slaves de cet espace, plusieurs types principaux peuvent être dégagés. Le premier est celui des grands personnages francs, chargés de la gestion, et notamment de la défense militaire dans ce secteur. Tel était notamment le cas de Cadolah qui, dans notre dossier, fut nommé pour la première fois en 818 en tant que "comte et préfet de la marche du Frioul "; il était déjà apparu auparavant dans d'autres sources latines, parce que dans la décennie précédente, il avait participé à un plaid en Istrie, dans lequel il avait arbitré, au nom de l'empereur, un conflit entre les habitants de la péninsule istrienne et leurs voisins slaves ${ }^{15}$. Le comte était ainsi considéré comme un connaisseur des questions slaves. C'est également à cette même catégorie que l'on peut rattacher son successeur Balderich. Celui-ci fut attesté pour la première fois en 815 en Saxe, chargé alors de diriger les alliés slaves des Francs contre les Danois $^{16}$; en 819 , il remplaça Cadolah en tant que dirigeant du Frioul, et s'illustra particulièrement dans la lutte contre les Slaves du sud. En revanche, il se montra moins efficace dans la lutte contre les Bulgares en 827, ce qui causa sa chute : il fut déposé par l'empereur en 828. Cette déchéance était significative : dans cet espace, Balderich fut l'un des premiers dirigeants francs qui, si l'on en croit les sources, fut puni par le pouvoir central pour une défaite subie face à agresseurs orientaux. Dans son cas comme dans celui de Cadolah, une des facettes principales de l'honneur était manifestement militaire.

Face à ces Francs se trouvait le chef slave Liudewit. Précisons d'emblée que celui-ci, malgré l'importance qu'il a acquise dans l'historiographie consacrée à l'Europe Centrale ${ }^{17}$, présente de nombreuses inconnues. Les seules données contemporaines à son propos proviennent des Annales du royaume des Francs, et encore n'est-ce que pour une période de cinq ans, entre 818 et la mort du personnage en $823^{18}$. L'imprécision est accrue par la mobilité géographique de ce chef : d'abord localisé à Siscia en Pannonie, Liudewit en fut chassé par les Francs, prit alors le pouvoir dans une cité située chez les Serbes, avant de revenir vers le nord-ouest dans la dernière année de son existence. A travers cet itinéraire aventureux, la principale constante que l'on puisse distinguer était le conflit militaire permanent qui l'opposa aux armées franques : parmi les chefs slaves de cet espace, Liudewit représentait celui qui ne voulait pas, ou ne pouvait pas s'intégrer dans la société carolingienne.

\footnotetext{
${ }^{14}$ Herwig Wolfram, Salzburg, Bayern, Österreich. Die Conversio Bagoariorum et Carantanorum und die Quellen ihrer Zeit, Vienne, 1995 (Mitteilungen des Instituts für Österreichische Geschichtsforschung 31), p. 159 sq.

${ }^{15}$ Les actes du plaid sont édités chez A. Petranović et A. Margetić, “Il Placito del Risano ”, Atti di centro di Ricerche Storiche - Rovigno 14 (1983-1984), p. 55-75. Parmi les études récentes consacrées à cet événement, on pourra consulter Stefan Esders, "Regionale Selbstbehauptung zwischen Byzanz und dem Frankenreich. Die Inquisitio der Rechtsgewohnheiten Istriens durch die Sendboten Karls des Grossen und Pippins von Italien ”, dans S. Esders et Th. Scharf éd., Eid und Wahrheitssuche. Studien zu rechtlichen Befragungspraktiken im Mittelalter und früher Neuzeit, Francfort/Main, 1999, p. 49-112, ou encore à Philippe Depreux, Les sociétés occidentales du milieu du VI à la fin du IX siècle, Rennes, 2002, p. 221 sq. et 293 sq., qui propose une traduction française de ce document.

${ }^{16}$ ARF, a. 815, p. 142. Pour les mentions ultérieures de ce personnage, voir le dossier de traductions proposé au terme de cet article.

${ }^{17}$ Cf. notamment, pour ne citer que deux ouvrages fort différents, Nada Klaić, Povijest Hrvata u ranom srednjem vijeku (L'histoire de la Croatie au Haut Moyen Age), Zagreb, 1971, ou plus récemment, Lujo Margetić, Dolazak Hrvata (La naissance de la Croatie), Split, 2001.

${ }^{18}$ Elles sont toutes regroupées dans le dossier de traductions proposé en fin du présent article.
} 
Entre ces deux premières catégories, on trouve enfin le cas de Borna, qui fut nommé surtout, dans les annales, pour les années 818 et 819. Là encore, il s'agissait d'un chef slave, qui ne fit lui aussi l'objet que de mentions sporadiques dans nos sources ${ }^{19}$, mais qui semble avoir été localisé dans un espace très proche du précédent. Mais par ses alliances diplomatiques, Borna adopta le parti opposé de celui de son voisin : il prit soin de se faire représenter lors des plaids tenus par les Carolingiens, et s'associa avec ces derniers dans le domaine militaire, ce qui l'amena notamment à entrer en conflit direct avec Liudewit. Ainsi, dans le jeu de définition perpétuelle des pouvoirs que constituait cet espace vers 820, Borna joua manifestement la carte franque.

Faut-il admettre que les conflits récurrents et importants entre ces groupes divers résultaient d'un clivage entre deux aires culturelles ? Souvent proposée, cette hypothèse postule implicitement une chronologie qui n'est pas évidente dans nos sources : le vide politique laissé par la disparition des Avars aurait engendré le développement progressif de nouveaux groupes ethniques à l'est des Francs, et ce seraient ces jeunes peuples qui, soucieux de préserver leur indépendance, auraient refusé toute intégration dans le monde franc et suscité ainsi des heurts fréquents sur les marges orientales du monde carolingien ${ }^{20}$. Dans ce schéma, Liudewit d'une part, les comtes francs du Frioul d'autre part n'auraient été que les jouets d'une évolution sociale, et leur rôle principal dans ces conflits aurait consisté à défendre les intérêts du groupe ethnique auquel ils appartenaient. Borna, quant à lui, aurait été une sorte d'intermédiaire indéterminé, parce qu'il était pris entre deux feux.

Cette interprétation culturelle ou ethnique a pour elle des arguments importants : on a signalé plus haut que la Pannonie et les Balkans constituaient bel et bien, vers 820, une aire de développement pour des sociétés nouvelles. Toutefois, un autre facteur d'explication transparaît dans les extraits proposés ici : il semble vraisemblable, en effet, que dans cette zone de conflits latents, les personnages que l'on vient de présenter avaient la possibilité d'atténuer, ou au contraire d'aggraver l'importance des tensions, et cela pour défendre leurs intérêts d'élite lorsque ceux-ci semblaient menacés.

Quels indices possédons-nous en ce sens ? Le premier d'entre eux réside dans les contradictions que présente le personnage de Liudewit. En effet, les sources, suivies en cela par les historiens modernes, décrivent le chef slave comme le principal adversaire militaire des Francs vers 820 ; mais à regarder les textes en détail, Liudewit ne prit jamais l'initiative d'un assaut militaire, se contentant de se retrancher dans des sites fortifiés, ou de prendre la fuite au rythme de la progression des armées carolingiennes. De la même façon, alors qu'il est présenté comme un rebelle vis-à-vis du pouvoir impérial, il annonça pourtant à plusieurs reprises (notamment en 822) son intention de se présenter devant Louis le Pieux pour se justifier. Un dernier point étonnant est la présentation par l'annaliste du déclenchement du conflit : on lit ainsi, pour l'année 818, que Liudewit accusait Cadolah lors d'un plaid, " parce que lui-même complotait quelque chose ". L'expression peut surprendre parce qu'à cette date, l'annaliste ne connaissait encore rien des intentions du chef slave, ni de la suite belliqueuse qu'allaient prendre les événements. On peut certes admettre une interpolation: si l'auteur écrivait a posteriori et connaissait ainsi le conflit des années ultérieures, il pouvait être tentant

\footnotetext{
${ }^{19}$ Les extraits les plus fiables concernant ce personnage, qui seront traduits ici, proviennent, là encore, des Annales du Royaume des Francs : c'est dans celles-ci que les auteurs médiévaux ultérieurs ont puisé leurs informations.

${ }^{20}$ Cette présentation des événements est proposée notamment par Harald Krahwinkler, Friaul im Frühmittelalter. Geschichte einer Region vom Ende des fünften bis zum Ende des zehnten Jahrhunderts, Vienne-Cologne-Weimar, 1992, p. 191, ou encore par Egon Boshof, Ludwig der Fromme, Darmstadt, 1996, p. 166.
} 
pour lui de l'annoncer par le biais de cette expression ${ }^{21}$. Mais une autre hypothèse semble également plausible : on peut également expliquer cette formule en considérant que celui qui était accusé par Liudewit en 818, à savoir le comte Cadolah, a pu juger nécessaire de discréditer son adversaire en le présentant comme un rebelle; et ce serait cette accusation, bien plus qu'une réelle révolte (non attestée) de Liudewit, qui aurait conduit aux opérations de représailles menées par les Francs dans les années suivantes. On s'expliquerait ainsi l'absence de réaction militaire du chef pannonien face aux armées franques, et son souci de venir se justifier devant l'empereur.

Cette hypothèse oblige à examiner l'un des principaux facteurs pour la constitution ou pour le déclin des élites, à savoir la circulation des informations entre le sud-est du monde franc et le pouvoir central. De quels renseignements l'autorité impériale disposait-elle pour déterminer quelle était la politique à suivre sur les marges orientales et, par conséquent, quelles étaient les élites locales à soutenir? A plusieurs reprises, dans les textes du début du $\mathrm{IX}^{\mathrm{e}}$ siècle, on est surpris de constater l'imprécision ou les lacunes dont souffraient les données disponibles à ce propos à la cour franque. Cette méconnaissance commence par la localisation des événements : il n'est ainsi pas rare de voir l'annaliste employer un même nom de peuple pour désigner, à quelques années d'intervalle, des régions séparées par plusieurs milliers de kilomètres ${ }^{22}$. Il est vrai que ces phénomènes concernaient généralement des peuples éloignés, qui ne présentaient pour les Francs qu'un intérêt limité ; mais on retrouve également la même ignorance à propos des Bulgares, qui constituaient quant à eux un vis-à-vis immédiat, et d'une importance stratégique majeure. Comment ces incertitudes se manifestent-elles dans notre dossier ? Entre 824 et 827, les Bulgares envoyèrent à plusieurs reprises des ambassadeurs vers Louis le Pieux pour négocier un tracé de frontières. De manière récurrente, l'empereur ignora leur requête, ce qui conduisit à une rupture diplomatique en 827 et à une défaite cruelle pour les Francs. La raison des hésitations impériales, selon l'annaliste, était qu' " une rumeur disait que le roi des Bulgares avait été chassé de son royaume, ou tué, par un des grands de son royaume". Si cette information avait été vérifiée, elle aurait considérablement modifié la donne diplomatique, et c'est pourquoi Louis le Pieux tergiversait, au risque de provoquer un conflit. Autrement dit, durant trois ans, personne à la cour franque ne fut en mesure de déterminer l'identité ou même l'existence de celui qui régnait sur les Bulgares, alors qu'il s'agissait là d'une des forces dominantes en Europe centrale : pour décider de l'attitude à adopter sur les marges orientales, le pouvoir central manquait cruellement d'informations.

Dans ces conditions, le monopole du renseignement revenait aux agents francs présents sur place. Ce fut ainsi vers Balderich que l'on se tourna lors de l'affaire bulgare, dans l'espoir d'en apprendre davantage ${ }^{23}$; de même, en 819 , Borna avait eu le privilège, selon une indication explicite de l'annaliste, d'être le principal témoin à la cour pour rapporter ses propres exploits au service des Francs. On peut constater ainsi que, de manière systématique, ceux que nos sources présentaient comme des alliés étaient avant tout ceux qui étaient en mesure de plaider leur cause auprès de l'oreille impériale ; inversement, ceux que l'annaliste décrivait comme des adversaires étaient précisément ceux qui ne pouvaient pas faire représenter leur point de vue devant le souverain. Compte tenu de ce jeu d'information et de désinformation, le comportement contradictoire de Liudewit pourrait s'expliquer non pas tant par la révolte d'un jeune peuple contre les Francs, mais par une mise en scène opérée par ses

\footnotetext{
${ }^{21}$ On sait que les Annales du Royaume des Francs firent l'objet de plus d'une retouche après leur rédaction originelle : Roger Collins, “ The Reviser Revisited : Another Look at the Alternative Version of the Annales Regni Francorum ”, dans Alexander C. Murray dir., After Rome's Fall. Narrators and Sources of Early Medieval History. Essays presented to W. Goffart, Toronto, 1998, p. 191-213. Mais ces interpolations portaient généralement sur la période antérieure à l'année 801 .

${ }_{22}$ Ainsi, l'auteur des Annales du Royaume des Francs, évoquant les Abodrites, localisait successivement ceux-ci au nord de la Bohême (ARF, a. 822, p. 159), puis “en Dacie, près du Danube” (ARF, a. 824, p. 165).

${ }^{23}$ Cf. l'extrait concernant l'année 826 , traduit plus loin dans le présent article.
} 
adversaires qui, contrairement à lui, parvenaient à se faire entendre à la cour. Cadolah, Borna et Balderich semblent avoir, sinon inventé de toutes pièces, du moins amplifié l'importance du conflit avec le chef pannonien pour sauvegarder leurs propres intérêts.

Il reste à expliquer les motivations de cette mise en scène, et l'on en vient ainsi à rechercher enfin quels étaient les intérêts de ces élites, et en quoi Liudewit pouvait les menacer. Pour répondre à cette question, il faut se souvenir que le statut social de ces groupes, loin d'être garanti par les institutions, devait être validé par un consensus : pour acquérir et préserver celui-ci, il fallait pouvoir présenter sa fonction comme indispensable pour le corps social. Or quels étaient les arguments que pouvaient avancer Borna ou Balderich pour justifier leur place au sein des élites franques?

Pour sa part, le chef slave Borna était inconnu à la cour carolingienne avant 818. Lors de sa première mention à cette date, il se présenta comme le chef de deux peuples slaves, les Timociani et les Guduscani ; et l'année suivante, d'après son propre récit, ce fut cette fonction qui lui permit de résister tant bien que mal aux assauts de Liudewit, et d'infliger quelques dommages à ce dernier. Borna se campait ainsi en chef d'un espace-tampon, qui offrait aux Francs un contrepoint utile face aux Slaves les plus belliqueux.

Cette présentation, toutefois, semble peu plausible, pour deux raisons au moins. La première d'entre elles tient à la localisation des peuples sur lesquels Borna affirmait régner. D'après l'hypothèse la plus généralement admise, le terme de Guduscani proviendrait du nom du fleuve qui s'appelle aujourd'hui la Gačka, sur la côte dalmate ${ }^{24}$; le terme de Timociani, quant à lui, semble devoir être relié au nom du Timok, affluent du Danube à l'est des Portes de Fer. Or ces deux espaces sont éloignés de plus de 500 kilomètres. Etant donné l'extrême morcellement des sociétés balkaniques au début du $\mathrm{IX}^{\mathrm{e}}$ s., il semble fort peu vraisemblable que Borna ait réellement régné sur deux peuples aussi éloignés. On pourrait imputer cette invraisemblance à une erreur de l'annaliste ; mais une autre interprétation est possible, si l'on prend en compte le fait qu'une telle exagération était profitable à ce prince en lui attribuant plus de pouvoir : on peut ainsi supposer que ce dernier, en arrivant à la cour franque, profita de la méconnaissance de ses auditeurs à propos du domaine slave pour s'attribuer plus d'importance qu'il n'en avait réellement, et accroître ainsi son rang dans la société carolingienne. Cette hypothèse semble d'ailleurs confirmée par l'attitude adoptée l'année suivante, en 819, par les peuples sur lesquels Borna affirmait régner : les Timociani prirent immédiatement le parti de Liudewit sans qu'on n'entende plus parler d'eux par la suite, tandis que les Guduscani firent provisoirement défection. A ce stade, Borna, pour lequel ces deux groupes constituaient auparavant une efficace carte de visite, n'était plus en mesure de faire valoir son rang au-dessus d'un peuple, ni son utilité pour les Francs. Certes, son pouvoir fut partiellement restauré l'année suivante, mais étant donné cette fragilité manifeste, on comprend mieux l'hostilité de son discours envers Liudewit: ce dernier disposait en effet, quant à lui, de forces militaires efficaces et, s'il avait pu se faire entendre auprès de la cour impériale, aurait probablement été mieux reçu que Borna lui-même, parce qu'il aurait constitué un allié plus intéressant. En désignant Liudewit comme un adversaire pour les Francs, Borna éliminait avant tout un concurrent dangereux pour lui-même.

Peut-on, de la même façon, considérer que l'attitude de Balderich dans ce conflit fut déterminée par la défense de ses propres intérêts sociaux ? Le comte, on s'en souvient, devait son rang avant tout à sa fonction d'homme de guerre. En ce sens, le statut qu'il pouvait revendiquer dans la société franque dépendait avant tout de l'existence d'un adversaire militaire considérable. En effet, les sources franques fournissent à volonté des cas de combattants dont les privilèges, loin d'être fixés une fois pour toutes, étaient négociés sans

\footnotetext{
${ }^{24}$ W. Pohl, Die Awaren..., op. cit., p. 264, avec la note 25.
} 
cesse, à la fois en fonction des qualités militaires de l'intéressé et de l'importance du danger auquel il faisait face ${ }^{25}$. En ce sens, si l'on admet que Liudewit ne nourrissait aucun projet de révolte, c'était une valeur ajoutée qui disparaissait pour ces combattants, Balderich en tête : une éventuelle réconciliation entre le chef slave et l'empereur allait ainsi à l'encontre des intérêts du comte de Frioul. On s'explique mieux ainsi les motivations du Franc pour attribuer, lui aussi, des projets de rébellion à Liudewit, et le refus de ce dernier de négocier avec ce même Balderich. Celui-ci, en effet, comme Borna avant lui, avait le souci de préserver son rang dans la société franque, même si la situation militaire ne l'exigeait plus.

Les élites dont il fut question ici profitaient ainsi d'un double écart social, à la fois vis-à-vis des catégories sociales qu'elles dominaient et de leurs propres maîtres. D'une part, en effet, les groupes slaves que l'on dénonçait ou que l'on contrôlait permettaient d'endosser le rôle d'un chef local ou régional; d'autre part, l'écart géographique entre la cour carolingienne et les agents francs des marges orientales conférait à ceux-ci un monopole de fait, qui faisait d'eux des intermédiaires indispensables. Ces groupes avaient ainsi tout intérêt à privilégier une double distinction, à la fois vers le haut et vers le bas; dans cette situation acrobatique, le danger à éviter résidait dans un rapprochement entre ces deux extrémités de la pyramide sociale, en l'occurrence entre le groupe slave de Liudewit et l'autorité impériale. Si l'on considère ces intérêts, et si l'on prend en compte les mécanismes de circulation des informations dans la société carolingienne, on gagne un facteur d'explication supplémentaire à propos des conflits entre Slaves et Francs : de part et d'autre existaient des élites de la guerre, pour lesquelles la pire crise aurait été la paix.

\section{Traduction des principales sources exploitées : extraits des Annales du Royaume des} Francs, 818-828 :

\section{a. 818 :}

L'empereur, passant par Rouen, Amiens et Cambrai, retourna à Aix-la-Chapelle pour y passer l'hiver (...).Vinrent là des légats d'autres nations, notamment des Abodrites et de Borna, duc des Guduscani ainsi que des Timociani, qui s'étaient naguère détournés d'une alliance avec les Bulgares et s'étaient tournés vers nos frontières; il y avait aussi [des envoyés] de Liudewit, chef de Pannonie inférieure, qui, parce qu'il complotait quelque chose, s'efforçait de faire passer pour cruel Cadolah, comte et préfet de la marche du Frioul (...).

\section{a. 819 :}

En juillet, un nouveau rassemblement eut lieu à Ingelheim, et une armée fut envoyée depuis l'Italie vers la Pannonie à cause de la révolte de Liudewit. Celle-ci revint sans grand succès, la situation étant presque pire qu'auparavant. Et Liudewit, gonflé d'orgueil, envoya des émissaires vers l'empereur, faisant mine de demander la paix, et avançant les conditions auxquelles il était prêt à exécuter ce qui lui serait ordonné. L'empereur n'accepta pas ces conditions et lui en proposa d'autres par l'intermédiaire de ses envoyés; de ce fait, Liudewit préféra s'obstiner dans la perfidie qu'il avait manifestée jusque-là, et il envoya ses légats vers les peuples voisins pour s'assurer leur appui dans la guerre. En particulier, il détourna le peuple des Timociani, qui s'était pourtant détourné d'une alliance avec les Bulgares et souhaitait se soumettre à l'autorité de l'empereur : par de fausses promesses, il parvint à lui faire oublier ce projet pour qu'il partage et soutienne sa propre perfidie.

(...)

Mais Borna, chef de Dalmatie, marcha, à la tête d'une grande armée, contre Liudewit qui se dirigeait vers lui ; il [Borna] fut abandonné par les Guduscani dès le premier choc, mais grâce à la protection de ses prétoriens, il put s'enfuir (...). Rentrés chez eux, les Guduscani furent à nouveau

\footnotetext{
${ }^{25}$ Ainsi, Dagobert avait exempté les Saxons de payer un tribut, parce que ceux-ci s'engageaient à défendre le royaume des Francs contre les Slaves de Samo, alors particulièrement menaçants : Frédégaire, Chronique (cf. plus haut, p. 2, note 10), livre 4, c. 74, p. 158.
} 
soumis par Borna. Mais exploitant les circonstances, Liudewit attaqua la Dalmatie en décembre avec une armée nombreuse, et la dévasta complètement, par le fer et par le feu. Borna, qui se sentait en position d'infériorité, enferma tout ce qu'il possédait dans ses places fortes et, avec des troupes sélectionnées, harcela Liudewit tantôt sur les flancs, tantôt dans le dos, de jour comme de nuit, et ne lui permit pas de retourner chez lui sans encombre. En définitive, il le contraignit au départ, après lui avoir infligé une perte de trois mille hommes et la capture de trois cents chevaux au moins, sans compter les bagages et toute sorte de butin. Le déroulement de ces opérations, il le fit raconter à l'empereur par l'intermédiaire de ses envoyés.

\section{a. 820 :}

L'hiver fini, dès qu'il y eut suffisamment d'herbe pour faire paitre les chevaux, on envoie trois armées contre Liudewit. L'une partit de l'Italie et passa par les Alpes noriques, l'autre par la province des Carinthiens, la troisième par la Bavière et la Pannonie supérieure ; et deux d'entre elles, à savoir celle de gauche et celle de droite, parvinrent au but avec du retard, parce que l'une fut empêchée de traverser les Alpes parce qu'une troupe d'adversaires s'y opposait, et que l'autre fut handicapée par la longueur du chemin et par l'existence du Danube qu'il fallait traverser ; quant à celle du milieu, qui était entrée par la Carinthie, elle traversa la Drave et ce fut elle qui parvint au but le plus rapidement, même si elle rencontra des résistances en trois endroits : elle eut plus de chance que les autres et triompha trois fois de l'ennemi. Liudewit, sans rien entreprendre contre elle, se contenta de se mettre à l'abri, lui et les siens, dans une place forte qu'il avait construite sur une montagne abrupte, et ni par la paix, ni par la guerre, on ne put l'amener à dialoguer, ni en personne ni par l'intermédiaire de légats, avec cette armée. Mais les trois armées, après s'être réunifiées, dévastèrent presque tout le pays par le feu et par le fer et rentrèrent chez elles sans avoir subi le moindre dommage. Seule celle qui était passée par la Pannonie supérieure fut gravement affectée, lorsqu'elle traversa la Drave, par une diarrhée causée par l'insalubrité du lieu et de l'eau. Ces trois armées avaient été envoyées depuis la Saxe, la Francie orientale et l'Alémanie, ainsi que depuis la Bavière et l'Italie. Lorsqu'elles furent rentrées, les habitants de la Carniole (ils habitent près de la Save et sont presque les voisins des Frioulans) se rendirent à Balderich ; et une partie des Carinthiens, qui nous avaient fait défection pour s'allier avec Liudewit, en firent de même.

\section{a. 822 :}

Une armée fut envoyée depuis l'Italie vers la Pannonie, pour mettre un terme au conflit contre Liudewit. Celui-ci, lorsque l'armée arriva, quitta la cité de Siscia ${ }^{26}$ et s'enfuit auprès des Serbes (on dit que ce peuple possède une grande part de la Dalmatie); ayant tué par ruse l'un de leurs chefs qui l'avait accueilli, il soumit la cité de ce dernier à son pouvoir. Il envoya pourtant des légats à l'empereur pour promettre qu'il se présenterait devant lui.

\section{a. 823 :}

[Lors d'une assemblée à Francfort,] l'empereur fut informé de la mort de Liudewit : celui-ci aurait quitté les Serbes, aurait gagné la Dalmatie et rejoint Liudemuhsl, l'oncle de Borna ; ayant passé quelque temps avec ce dernier, il aurait été tué par une ruse de celui-ci.

\section{a. 825 :}

L'empereur, revenu de la chasse, accueillit une ambassade des Bulgares ${ }^{27}$; il s'agissait de définir les frontières entre Francs et Bulgares.

\section{a. 826 :}

Lorsque les légats bulgares firent leur rapport auprès de leur roi, celui-ci envoya une nouvelle fois des lettres vers l'empereur et lui demanda de régler sans plus attendre la question des frontières ; autrement, chaque camp n'aurait qu'à défendre ses frontières, sans passer par un traité de paix. Mais l'empereur ne répondit pas tout de suite, parce qu'une rumeur disait que le roi des Bulgares avait été chassé de son royaume, ou tué, par un des grands de son royaume; ordonnant à l'ambassadeur de

\footnotetext{
26 Aujourd'hui Sisak, en Croatie.

${ }^{27}$ Ceux-ci avaient déjà essayé à plusieurs reprises d'entrer en contact avec Louis le Pieux en 824.
} 
patienter, il envoya Bertrich, comte du palais, auprès de Balderich, de Gerold et des gardiens de la frontière qui, en Carinthie, faisait face aux Avars : cela pour savoir si la rumeur disait vrai. Bertrich ne rapporta rien de sûr à ce sujet : c'est pourquoi l'empereur fit rentrer l'ambassadeur chez lui sans lui confier de lettre.

(...) Les comtes Balderich et Gerold, ainsi que les préfets de la marche de Pannonie, participèrent à un plaid et affirmèrent qu'ils n'avaient rien remarqué à propos d'un mouvement des Bulgares contre nous.

\section{a. 827 :}

Les Bulgares remontèrent la Drave avec une armée navale, dévastèrent par le fer et par le feu les Slaves qui habitaient en Pannonie et, ayant chassé les chefs de ces derniers, placèrent des dirigeants bulgares à leur tête.

\section{a. 828 :}

Le duc Balderich du Frioul, dont la mollesse avait permis à l'armée des Bulgares de dévaster impunément les frontières de la Pannonie supérieure, fut privé de ses honneurs; et la marche qu'il était seul à posséder fut divisée entre quatre comtes. 